\title{
A novel bandwidth efficient technique for ICI cancellation in OFDM system
}

\author{
Alka Kalra $^{1 *}$ and Rajesh Khanna ${ }^{2}$ \\ ${ }^{1}$ Department of Electronics and Communication Engineering Haryana College of Technology and Management, Kaithal \\ Haryana, India. \\ ${ }^{2}$ Department of Electronics and Communication Engineering, Thapar University, Patiala, Punjab, India.
}

Accepted 14 May, 2012

\begin{abstract}
A well-known problem of orthogonal frequency division multiplexing (OFDM) is its sensitivity to offset between the transmitted and received carrier frequencies. In OFDM communication systems, the frequency offsets in mobile radio channels deform the orthogonality between subcarriers which causes inter carrier interference (ICl). $\mathrm{ICl}$ causes power leakage among subcarriers and it further degrades the system performance. In this paper a novel bandwidth efficient method for combating the effects of ICl is presented. In the present scheme, the self cancellation mechanism is used to compress ICl signals without any equalization. At transmitter and receiver parallel Fast Fourier Transform (FFT) factorization is performed using Radix-2 decimation in frequency (DIF) FFT algorithm derived from the well known Cooley-turkey factorization. The proposed scheme cancels the ICl coefficient effectively and further improves system performance as shown through extensive simulations.
\end{abstract}

Key words: Orthogonal frequency division multiplexing (OFDM), intercarrier interference, carrier frequency offset, Radix2, fast Fourier transform (FFT).

\section{INTRODUCTION}

Orthogonal frequency division multiplexing has gained considerable attention due to its high speed data applications. It is emerging as most preferred digital modulation technique due to its high bandwidth efficiency and strong ability to handle multipath interference. In an OFDM system, the whole available bandwidth is divided into $\mathrm{N}$ parallel streams, and a block of $\mathrm{N}$ data symbols are modulated on $\mathrm{N}$ corresponding subcarriers which are orthogonal to each other. The spectra of the subcarriers are overlapping; therefore precise frequency recovering is needed. However, in the mobile radio environment, the relative movement between transmitter and receiver causes Doppler frequency shift, in addition the carriers can never be perfectly synchronized. These random frequency errors in OFDM system distort orthogonality between subcarriers, as a result intercarrier interference (ICl) occurs. Literature shows that in such systems, the bit error rate (BER) increases rapidly with increasing frequency offsets (Pollet et al., 1993). Therefore, ICI has negative impact on data throughput. Researchers have proposed several methods to reduce ICI. The first approach is frequency-domain equalization in which weighting coefficients of equalizer need be chosen by channel state information (CSI) estimation (Ahn and Lee, 1993). The second one is time-domain windowing (Muschallik, 1996). The third approach is ICl self cancellation, in which redundant data is transmitted onto adjacent subcarriers such that the $\mathrm{ICl}$ between adjacent sub carriers cancels out at the receiver (Zhao et al., 1998). This paper is focused on ICl-self-cancellation technique. There are various self-cancellation techniques with different $\mathrm{ICl}$ cancelling modulation methods and the corresponding demodulation methods proposed to reduce the $\mathrm{ICl}$ caused by carrier frequency offset (CFO). 

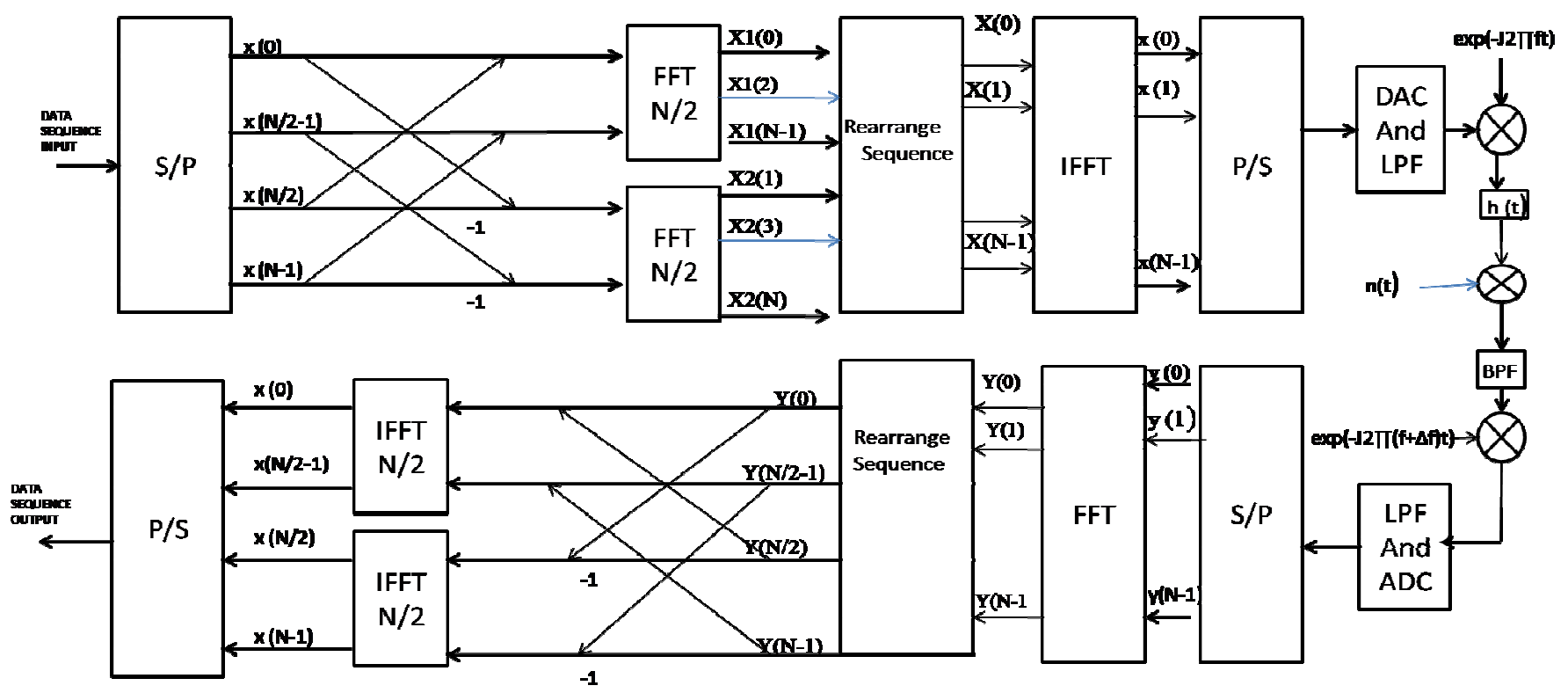

Figure 1. Bandwidth efficient model for ICI Suppression using Radix-2.

These methods include the adjacent data-conversion method (Zhao and Haggman, 2001), the symmetric dataconversion method (Sathananthan and Tellambura, 2001), the adjacent data-conjugate method and the symmetric data-conjugate method (Sathananthan et al., 2004). The aforementioned schemes can suppress the $\mathrm{ICl}$ effectively but the spectral efficiency will obviously be reduced as data is replicated on two or more subcarriers.

However, to increase spectral efficiency various schemes has been proposed in literature. DFT based bandwidth efficient scheme for $\mathrm{ICl}$ cancellation was proposed by Bing Han et al. (2003). In this scheme at the transmitter side, before modulated onto a group of adjacent subcarriers, the parallel data symbols are divided into two groups, each discrete Fourier transformed. At receiver side, after multicarrier demodulation, the received symbols are divided into two groups again and each inverse-discrete-Fourier transformed respectively to rebuild the transmitted symbols. However, this scheme outperforms only for small frequency offset values $(\varepsilon<0.2)$. In this direction another scheme based on two path algorithm (TPA) was proposed by Kamboj et al. (2009). The first path sends a specially modulated data symbols which results from weighted subtraction of an even numbered modulated symbol and its consecutive symbol. The second path uses the conjugate of a similar type of specially modulated data symbols which results from weighted addition of an even numbered modulated symbol and its consecutive symbol. With additional signal processing, bandwidth efficiency of system can be achieved. A new bandwidth efficient $\mathrm{ICl}$ cancellation scheme to cancel interference in OFDM system was proposed by equalization of interference coefficients without requiring any training data (Kumar and Pandey, 2009).
In this paper, a new bandwidth efficient scheme is proposed in which parallel Fast Fourier Transform factorization is performed using Radix -2 DIF FFT algorithms derived from the well known Cooley-turkey factorization using butterfly algorithm. The DIF radix-2

FFT partitions the DFT computations into even indexed and odd indexed outputs which can be computed by shorter length DFT's of different combinations of input samples. The proposed scheme cancels the $\mathrm{ICI}$ coefficients and improves system performance of OFDM system. The paper is organized as: The system model of the present scheme is shown. Discussion of the simulation results for the proposed scheme and a comparison with previous bandwidth efficient schemes is further presented. Finally, the overall findings of the study are summarized.

\section{SYSTEM MODEL AND DESCRIPTION}

In this section, we propose bandwidth efficient model for $\mathrm{ICl}$ cancellation in OFDM system as shown in Figure 1. At transmitter each block of $\mathrm{N}$ information symbols are converted from serial to parallel form as sequence of vectors $\mathrm{X}=\left[\mathrm{X}_{0}, \mathrm{X}_{1} \ldots \ldots \mathrm{X}_{\mathrm{N}-1}\right]$, where $\mathrm{N}$ is assumed to be equal to IDFT length of OFDM modulator and its integer power of two. DIF FFT algorithm decomposes the DFT by recursively splitting the sequence elements $X(k)$ in frequency domain into sets of smaller subsequences as

$$
\begin{aligned}
& X_{1}(k)=\sum_{n=0}^{\frac{N}{2}-1}\left[X(n)+X\left(n+\frac{N}{2}\right)\right] W_{N / 2}^{n} \\
& X_{2}(k)=\sum_{n=0}^{\frac{N}{2}-1}\left[X(n)-X\left(n+\frac{N}{2}\right)\right] W_{N}^{n} W_{N / 2}^{n}
\end{aligned}
$$



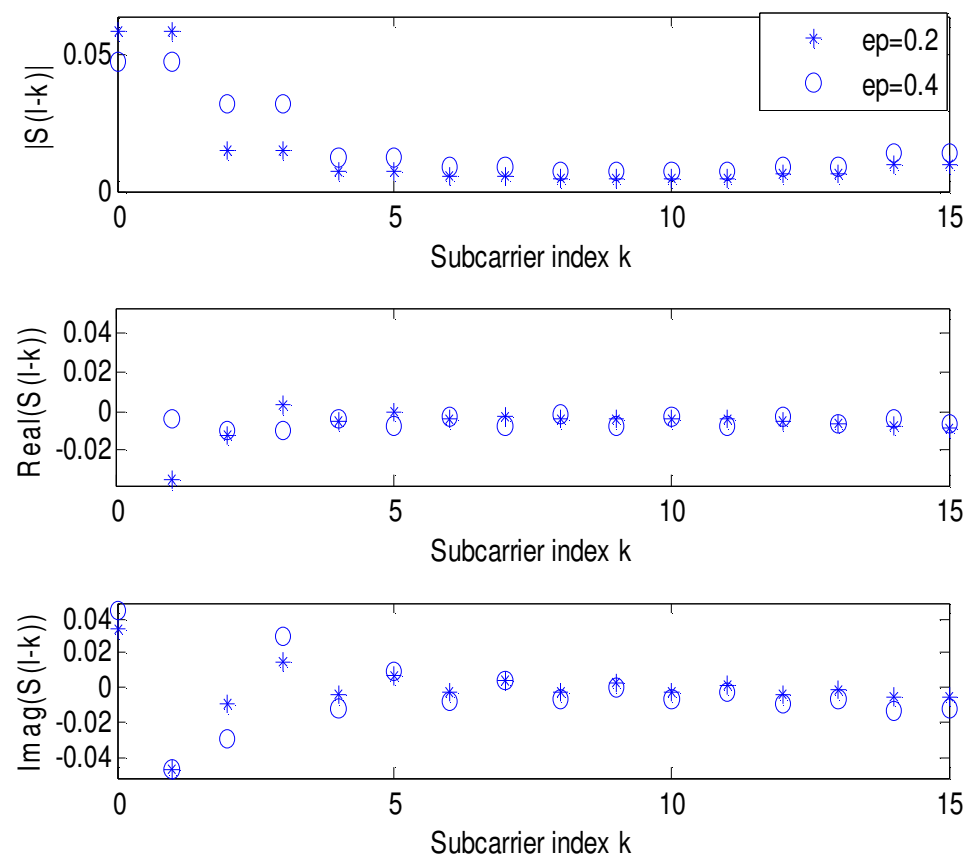

Figure 2. a) Amplitude of $S(l-k)$; b) Real part of $S(l-k)$; c)
Imaginary part of $S(l-k)$.

Let $X(k)$ be combined effect of reordered sequence of $X_{1}(k)$ and $X_{2}(k)$. Inverse Fourier transform (IFFT) is applied on $X(k)$.The received signal at subcarrier $\mathrm{k}$ is represented as

$x(n)=\frac{1}{N} \sum_{k=0}^{N-1} X(k) W_{N}^{-n k} \exp ^{\frac{j 2 \pi \varepsilon n}{N}}+w(n)$

We have assumed that signal is transmitted in additive white Gaussian noise (AWGN) channel and received signal is only affected by carrier frequency offset (CFO) error. Let $\varepsilon$ is normalized frequency offset $\Delta f . N T_{\mathrm{s}}$, where $\Delta f$ is the carrier-frequency offset of the local oscillators between the transmitter and the receiver and $T$ s denotes the subcarrier symbol period. The effect of this frequency offset on the received symbol stream can be understood by considering the received symbol $Y(k)$ on the $k^{\text {th }}$ subcarrier. In the following analysis, we will not consider the guard interval and assume that there is no overlap between different OFDM symbols. The received signal at subcarrier index $k$ can be expressed as:

$$
\begin{gathered}
Y(k)=\sum_{l=0}^{N-1} x(n) e^{\frac{-j 2 \pi k n}{N}} \\
=X(k) S(0)+\sum_{\substack{l=0 \\
l \neq k}}^{N-1} X(l) S(l-k)+W(k)
\end{gathered}
$$

On right hand side of above equation $(I)$ is desired sequence, $(I I)$ is $\mathrm{ICl}$ and $(I I I)$ is AWGN noise introduced in the channel. Let $X(l)$ is transmitted symbol on $l^{\text {th }}$ subcarrier and sequence $S(l-k)$ is defined as the $\mathrm{ICl}$ co-efficient between $l^{\text {th }}$ and $k^{\text {th }}$ sub carrier which is expressed as

$$
S(l-k)=\frac{\sin [\pi(l+\varepsilon-k)]}{N \sin \left[\frac{\pi}{N}(l+\varepsilon-k)\right]} \exp ^{j \pi\left(1-\frac{1}{N}\right)(l+\varepsilon-k)}
$$

Without frequency error $\varepsilon=0,\left|S_{0}\right|$ takes its maximum value. It is evident that as $\varepsilon$ becomes larger, the desired part decreases and the undesired part increase.

In Figure 2 effect of $\mathrm{ICl}$ coefficients at receiver of proposed scheme is presented taking $\mathrm{N}=16$ at different offset values 0.2 and 0.4 respectively. The $\mathrm{ICl}$ coefficients are cancelled out with each other and its effect becomes consistent with increase in frequency offset. The proposed scheme reduces $\mathrm{ICl}$ component even at high frequency offset. Applying N/2 IFFT to received signal we have

$$
\begin{aligned}
x_{1}(k)=\frac{1}{N} \sum_{\substack{k=0 \\
l \neq k}}^{\frac{N}{2}-1}[Y(k)+ & \left.Y\left(k+\frac{N}{2}\right)\right] W_{\frac{N}{2}}^{-l k}+W_{k 1}^{\prime} \\
& \left.=\frac{1}{N} \sum_{k=0}^{\frac{N}{2}-1} \sum_{\substack{=0 \\
l \neq k}}^{N-1} X(K)\left[S(l-k)+S\left(l-k+\frac{N}{2}\right)\right]\right) W_{N}^{-l k}+W_{k 1}^{\prime}
\end{aligned}
$$




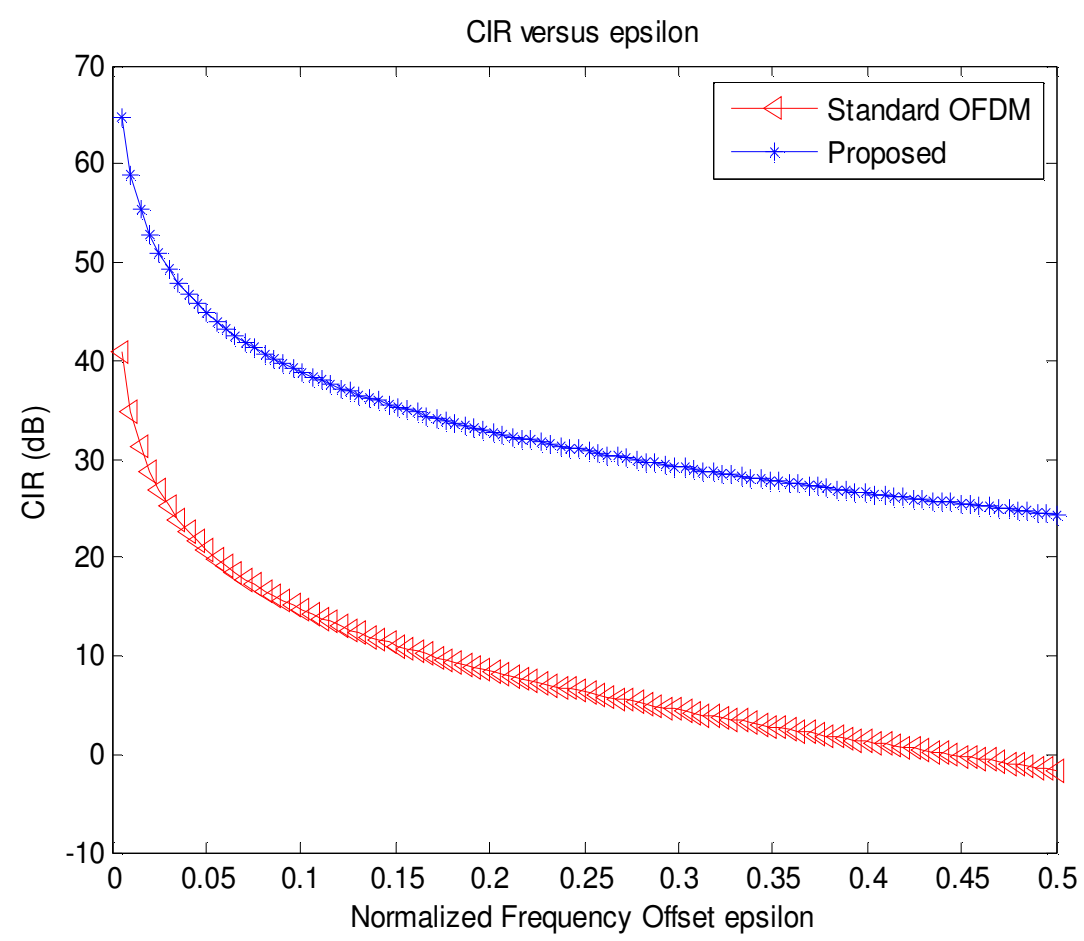

Figure 3. CIR comparison.

$$
\begin{gathered}
x_{2}(k)=\frac{1}{N} \sum_{k=0}^{\frac{N}{2}-1}\left[Y(k)-Y\left(k+\frac{N}{2}\right)\right] W_{\frac{N}{2}}^{-l k}+W_{k 2}^{\prime} \\
= \\
\sum_{k=0}^{\frac{N}{2}-1} \sum_{l \neq k}^{N-1} X(k)\left[S(l-k)-S\left(l-k+\frac{N}{2}\right)\right] W_{N}^{-l(k+1)}+ \\
W_{k 2}^{\prime}
\end{gathered}
$$

where $W_{k 1}^{\prime}$ and $W_{k 2}^{\prime}$ are N/2 point IFFT of noise samples. Applying symmetry and periodicity property in (5), the ICI coefficients will be zero for even subcarriers and $S(l-k)$ for odd subcarriers. Similarly from (6), ICI coefficients will be zero for odd subcarriers and $S(l-k)$ for even subcarriers. Thus overall effect of $\mathrm{ICI}$ is reduced at even symbols and odd symbols respectively.

\section{SIMULATION RESULTS AND DISCUSSION}

The simulations have been conducted for performance comparison of various bandwidth efficient techniques is used for $\mathrm{ICl}$ cancellation in OFDM system. Figure 3 shows significant improvement in carrier to interference ratio (CIR) of proposed system compared to standard OFDM system. We have performed simulations using MATLAB for BPSK/OFDM with 128 subcarriers. The normalized frequency offset is set to be $\varepsilon=0.1,0.3$ and 0.5 respectively. The performance of various schemes of $\mathrm{ICl}$ cancellation is compared on basis of bit error rate (BER). We have assumed ideal reception of OFDM symbols for all schemes. BER of standard OFDM, DFTOFDM, TPA algorithm and proposed (Radix-2) algorithm is plotted in Figure 4. It shows DFT-OFDM, TPA algorithm and present scheme has SNR gain of 8,6,13 $\mathrm{dB}$ respectively at BER of $10^{-2}$ for low frequency offset that is $\varepsilon=0.1$ than standard OFDM system. In Figure 5 , it can be observed DFT-OFDM and proposed algorithm has significant improvement in performance than other schemes at $\varepsilon=0.3$. The SNR gain of $10 \mathrm{~dB}, 5 \mathrm{~dB}, 13 \mathrm{~dB}$ is achieved at $10^{-2}$ for DFT-OFDM, TPA algorithm and proposed algorithm respectively over standard OFDM performance. From Figure 6 it can be observed clearly the proposed algorithm performs better as compared to another schemes in Rayleigh channel also. The proposed scheme outperforms than other bandwidth efficient algorithms at high frequency offset values. Moreover, spectral efficiency of proposed scheme is maintained without much increase in system complexity.

\section{CONCLUSION}

In this paper, ICl self-cancellation in OFDM systems has been investigated. The bandwidth efficiency for normal $\mathrm{ICl}$ self cancellation schemes suffers as redundant information is required for such schemes. In proposed Radix 2 based scheme repetition of data symbols and pilot insertion is not required. Thus bandwidth efficiency of system is maintained. At high frequency offset DFTOFDM, TPA scheme do not perform well. Through 


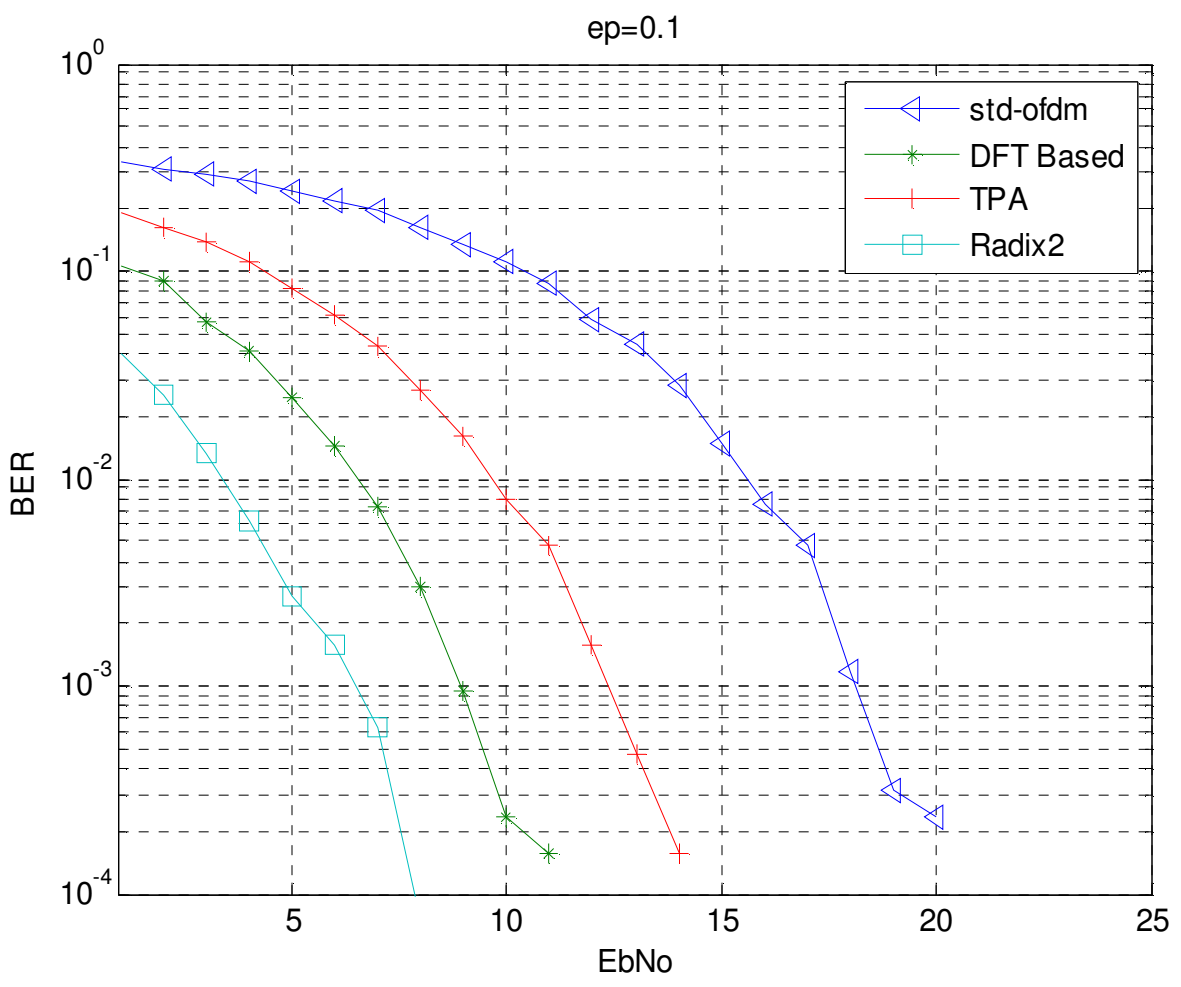

Figure 4. BER comparison with BPSK, $A W G N, \varepsilon=0.1$.

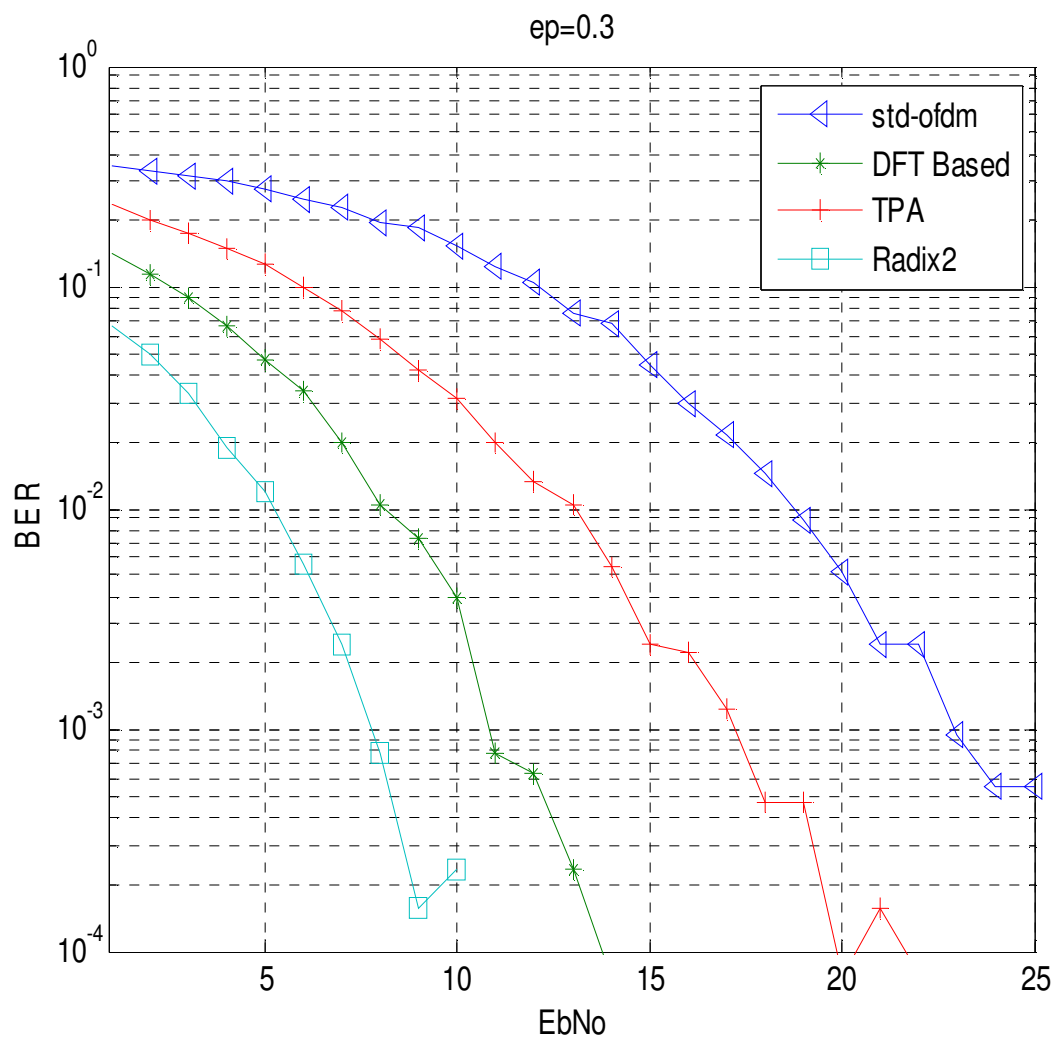

Figure 5. BER ccomparison with BPSK, AWGN Channel, $\varepsilon=0.3$. 


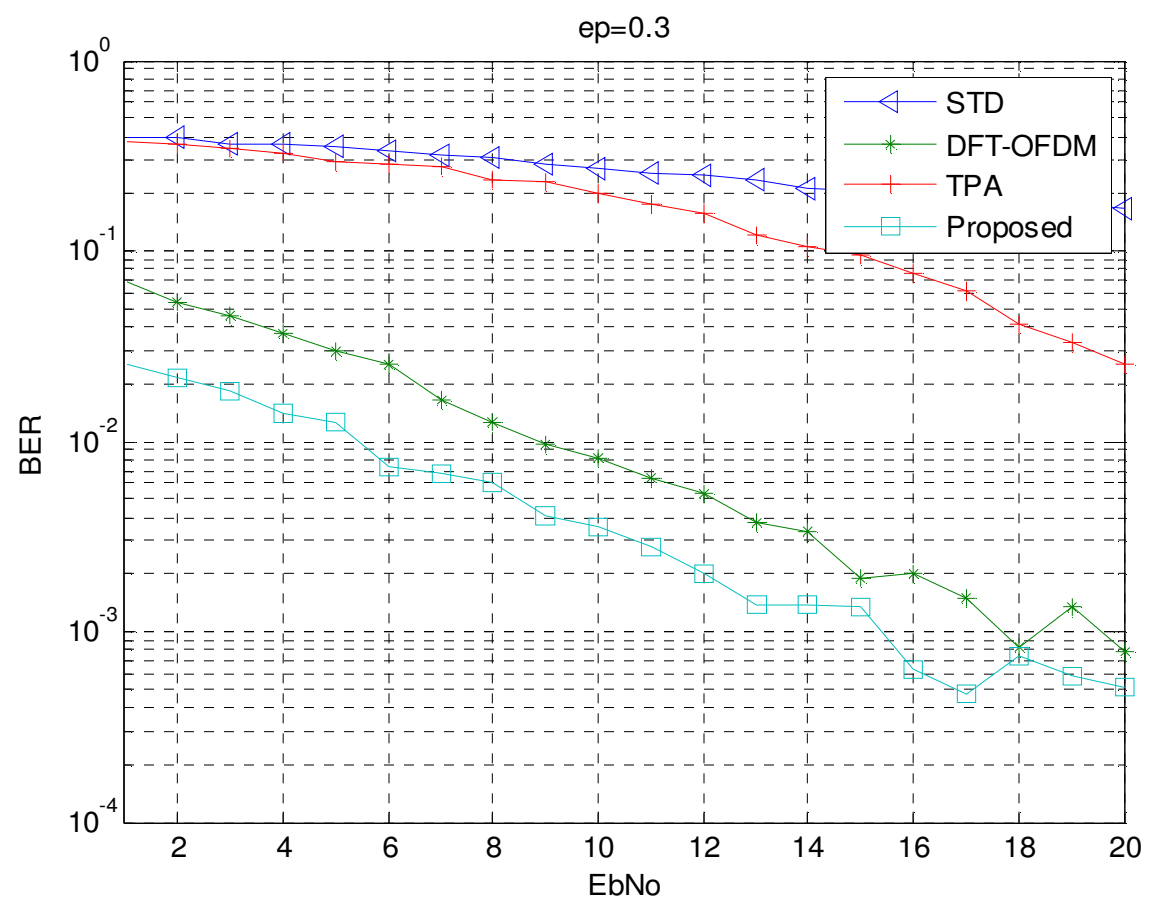

Figure 6. BER comparison with BPSK, Rayleigh Channel, $\varepsilon=0.3$.

simulations and system model it has been verified that proposed scheme mitigates $\mathrm{ICl}$ efficiently at even high frequency offset $(\varepsilon<0.4)$ without sacrificing system bandwidth efficiency. Since no equalization procedure is needed, the system can be significantly simplified.

\section{REFERENCES}

Ahn J, Lee HS (1993). Frequency domain equalization of OFDM signal over frequency nonselective Rayleigh fading channels. Electron. Lett. 29(16):1476-1477.

Han B, Gao X, You X, Costa E (2003). A DFT-based ICI Selfcancellation Scheme for OFDM Systems. Commun. Technol. Proc. ICCT. 2:1359-1362.

Kamboj A, Keshari A, Dwivedi VK, Singh G (2009). Bandwidth Efficient Intercarrier Interference Cancellation Technique for OFDM Digital Communication Systems. PIERS Proceedings, 5:1244-1248.

Kumar A, Pandey R (2009). A bandwidth-efficient method for cancellation of $\mathrm{ICl}$ in OFDM systems. Int. J. Electron. Commun. 63:569-575.

Muschallik C (1996). Improving an OFDM reception using an adaptive Nyquist windowing. IEEE Trans. Consum. Electron., 42:259-269.
Pollet T, Van Bladel M, Moeneciaey M (1993). BER sensitivity of OFDM system to carrier frequency offset and Wiener phase noise. IEEE Trans. Commun. 43:191-193.

Sathananthan K, Athaudage CRN, Qiu B (2004). A novel ICI cancellation scheme to reduce both frequency offset and IQ imbalance effects in OFDM. Proc. IEEE $9^{\text {th }}$ International Symposium on Computers and Communications, pp. 708-713.

Sathananthan K, Tellambura C (2001). Probability of error calculation of OFDM systems with frequency offset. IEEE Trans. Commun. 49(11): 1884-1888.

Zhao Y, Haggman SG (2001). Intercarrier Interference self-cancellation scheme for OFDM mobile communication systems. IEEE Trans. Commun., 49(7):1185-1191.

Zhao Y, Leclercq JD, Häggman SG (1998). Intercarrier interference compression in OFDM communication systems by using correlative coding. IEEE Commun. Lett. 2:214-216. 